\title{
Orbital degrees of freedom as origin of magnetoelectric coupling in magnetite
}

\author{
Kunihiko Yamauchi and Silvia Picozzi \\ Consiglio Nazionale delle Ricerche (CNR-SPIN), 67100 L'Aquila, Italy
}

(Dated: November 13, 2018)

\begin{abstract}
A microscopic understanding of magnetoelectricity, i.e. the coupling between magnetic (electric) properties and external electric (magnetic) fields, is a crucial milestone for future generations of electrically-controlled spintronic devices. Here, we focus on the first magnetoelectric known to mankind: magnetite. By means of a joint approach based on phenomenological Landau theory and density-functional simulations, we show that magnetoelectricity in charge-/orbital-ordered $\mathrm{Fe}_{3} \mathrm{O}_{4}$ in the non-centrosymmetric $C c$ structure is driven by the interplay between a peculiar orbital-order and on-site spin-orbit coupling. The excellent agreement with available experiments confirms our theoretical picture, pointing to magnetite as a prototype of a novel category of magnetoelectrics where ferroelectric polarization can be induced, tuned or switched via a magnetic field.
\end{abstract}

PACS numbers: Valid PACS appear here

Magnetoelectric (ME) effects - i.e. how to control magnetic (electric) properties via electric (magnetic) fields - have been intensively investigated in recent years, often in connection to multiferroics, materials where two or more ferroic properties (i.e. long range spontaneous magnetism, deformation or ferroelectricity) coexist in the same phase. [1-3] In this framework, it was recently found ferrimagnetic magnetite $\left(\mathrm{Fe}_{3} \mathrm{O}_{4}\right)$ to show a sizable ferroelectric (FE) polarization at low temperatures, with the microscopic mechanism clarified theoretically: [4, 5] the FE polarization, of the order of few $\mu \mathrm{C} / \mathrm{cm}^{2}$, is induced purely by the non-centrosymmetric charge-order (CO), a mechanism that is rarely seen in other materials. [6]

The ME effect in magnetite was discovered by Rado and Ferrari in 1975 9]; however, its importance has been neglected for long time. It is the aim of this paper to clarify the microscopic origin of this peculiar ME effect in magnetite, by means of Landau theory and densityfunctional-theory (DFT). We start from two important observations made in Ref. 9: (i) The electric polarization $\boldsymbol{P}$ behaves as a nonlinear function of the applied magnetic field $\boldsymbol{H}$, more precisely, $P_{a} \propto \sin ^{2} \theta$ (with negligible $\sin ^{4} \theta$ term), with $\theta$ denoting the polar angle of the $\boldsymbol{H}$ direction at $4.2 \mathrm{~K}$. (ii) The observed $P$ (reported in arbitrary units) is saturated with respect to a certain $H^{\text {sat }}$, i.e. its behavior being similar to the magnetization $M$. This implies that $\boldsymbol{P}$ arises in the perfectly "collinear" ferrimagnetic configuration, with all spins simultaneously following the direction of $\boldsymbol{H}$ (i.e. $\boldsymbol{M} / / \boldsymbol{H})$; this shows a distinct contrast with respect to many cases of singlephase magnetoelectrics, where either $\boldsymbol{H}$-induced "noncollinear" spin canting or spin spiral modulation occur (e.g. at $\mathrm{Cr}_{2} \mathrm{O}_{3}[10], \mathrm{LiNiPO}_{4}[11]$ and $\mathrm{TbMnO}_{3}[12]$ ).

Structural Details. - Magnetite shows a well-known metal-insulator transition, namely the "Verwey transition" at $T_{\mathrm{V}}=120 \mathrm{~K}$, below which the crystal structure changes from cubic $F d 3 m$ to a less symmetric structure; correspondingly, the $\mathrm{Fe}^{2+} / \mathrm{Fe}^{3+}$ charge ordering, observed on Fe-B sites in the inverse-spinel $\mathrm{AB}_{2} \mathrm{O}_{4}$ lattice, 13, 14] arises. Despite several differ-

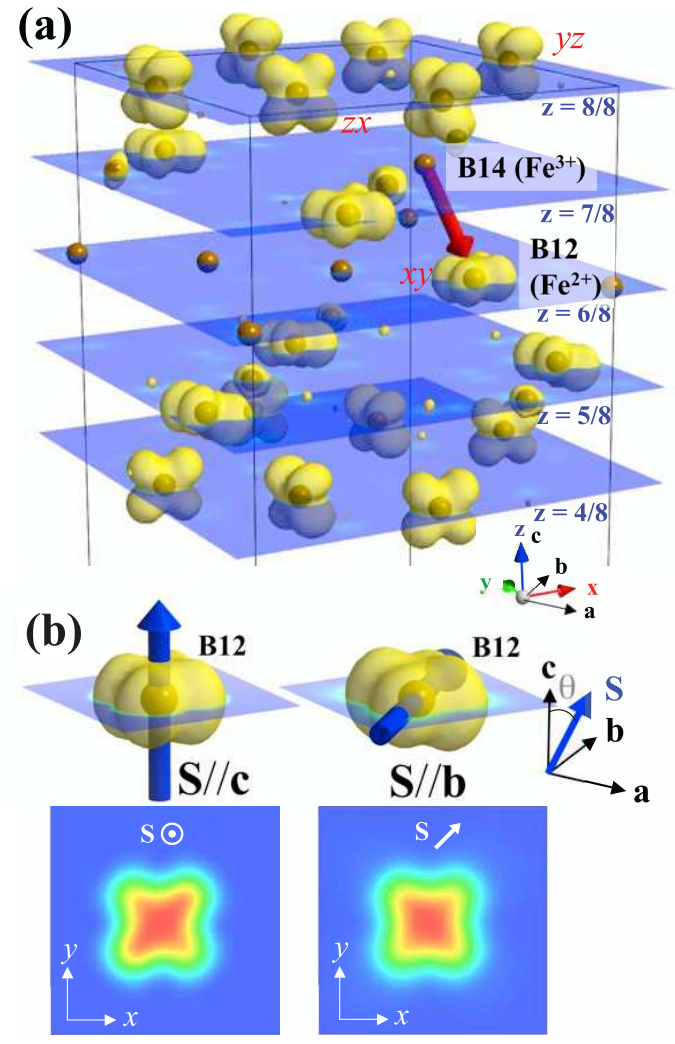

FIG. 1: (a) Charge/orbital ordering shown by an isosurface of charge density of Fe- $t_{2 g}^{\downarrow}$ states in the upper half $(1 / 2<z<1)$ of the primitive $C c$ unit cell (for details, see ref. 5). The red arrow shows the electric dipole connecting $\mathrm{B} 12\left(\mathrm{Fe}^{2+}\right)$ and $\mathrm{B} 14\left(\mathrm{Fe}^{3+}\right)$ sites, responsible for the ferroelectric $\boldsymbol{P}$. Vectors $(\boldsymbol{a}, \boldsymbol{b}, \boldsymbol{c})$ denote directions of the conventional lattice vectors, linked to the cartesian coordinate system through $\boldsymbol{x}=\boldsymbol{a}+\boldsymbol{b}$, $\boldsymbol{y}=-\boldsymbol{a}+\boldsymbol{b}$ and $\boldsymbol{z} \simeq \boldsymbol{c}$ (due to a small monoclinic distortion). The occupied orbital state at some $\mathrm{Fe}^{2+}$ sites is labeled as $x y / y z / z x$, used as site index hereafter. (b) Change of the charge density (an isosurface and a section) on Fe-B12 site upon rotation of the spin direction (shown by blue arrows in the upper panel). To emphasize the effect, SOC term is enhanced by a factor of 10 .

ent space groups were proposed for the low-temperature 
structure, 14] a base-centered monoclinic $C c$ symmetry (\#9) was suggested as ground state both by experimental and theoretical studies. [15, 16] The absence of inversion symmetry in the $C c$ space group and the consequent polar CO pattern were found to be relevant for the presence of ferroelectricity. [4] Indeed, the FE polarization is primarily caused by uncompensated local electric dipoles connecting $\mathrm{Fe}^{2+}$ and $\mathrm{Fe}^{3+}$ ions at $\mathrm{B}$ sites; as shown in Fig प(a) and deeply discussed in Refs. [5, 17], $\mathrm{Fe}^{2+}$ at B12 site and $\mathrm{Fe}^{3+}$ ions at B14 site form one of the dipoles responsible for the net $\boldsymbol{P}$ in the $C c$ unit cell. Fig:1(a) also shows the orbital order $(\mathrm{OO})$ at $\mathrm{Fe}^{2+}\left(d^{6}\right)$ sites. Being coupled to cooperative Jahn-Teller (JT) distortions, the partially filled minority-spin $t_{2 g}^{1 \downarrow}$ orbital shows one of the three $d_{x y} / d_{y z} / d_{z x}$ charge distributions. In the primitive unit cell, $32 \mathrm{Fe}-\mathrm{B}$ sites are split into $16 \mathrm{Fe}^{3+}$ sites and $16 \mathrm{Fe}^{2+}$ sites; in addition, the latter JT-active sites are furthermore split into three groups: $8 x y, 4 y z$ and 4 $z x$ sites. 18 The OO pattern is such that the orbitals avoid to overlap, consistent with reducing the inter-site Coulomb repulsion and optimizing cooperative JT distortions. Consequently, these orbitals do not fully lie in the $x y / y z / z x$ planes, but are rather slightly tilted.

Macroscopic Model. - Hereafter, we separate the spontaneous CO-induced, $\boldsymbol{P}^{\mathrm{CO}}$, and the $\boldsymbol{M}$-directiondependent, $\boldsymbol{P}^{\mathrm{ME}}(\boldsymbol{M})$. According to Ref. 19, the mechanisms leading to $\boldsymbol{P}^{\mathrm{ME}}$ are generally classified as driven by magnetostriction $\left(P^{\mathrm{MS}}\right)$, spin-current $\left(P^{\mathrm{sp}}\right)$ and orbital degrees of freedom $\left(P^{\text {orb }}\right)$. In our case, the collinear ferrimagnetic configuration rules out both $P^{\mathrm{MS}}$ and $P^{\mathrm{sp}}$, whereas only $P^{\text {orb }}$ can be induced by the spin-orbit coupling (SOC) within orbital-unquenched $t_{2 g}^{4} e_{g}^{2}$ state at $\mathrm{Fe}^{2+}\left(d^{6}\right)$ sites. Summarizing, the total polarization is here described as $\boldsymbol{P}^{\text {total }}(\boldsymbol{M})=\boldsymbol{P}^{\mathrm{CO}}+\boldsymbol{P}^{\text {orb }}(\boldsymbol{M})$. As we will discuss later, the $\boldsymbol{P}^{\text {orb }}$ term is deeply related to the single-site magnetic anisotropy energy (MAE), both having SOC as common underlying origin.

TABLE I: Matrices of the generators of the $C c$ space group in the representations spanned by $M$ and $P$. The space group elements are denoted as the identity $E$ and $c$-glide $=\left\{\sigma_{b} \mid 00 \frac{1}{2}\right\}$, with time reversal $T$.

\begin{tabular}{|c|cccc||c|cccc|}
\hline & $E$ & $c$ & $T E$ & $T c$ & & $E$ & $c$ & $T E$ & $T c$ \\
\hline$M_{a}, M_{c}$ & 1 & -1 & -1 & 1 & $P_{a}, P_{c}$ & 1 & 1 & 1 & 1 \\
$M_{b}$ & 1 & 1 & -1 & -1 & $P_{b}$ & 1 & -1 & 1 & -1 \\
\hline
\end{tabular}

In order to shed light on the peculiar ME effects in $\boldsymbol{P}^{\text {orb }}$, we briefly show the group theory analysis and a derivation based on the Landau theory of phase transitions. 20] When working in the $C c$ space group with the symmetry operations $\{E, c\}$, the ferrimagnetic order leads to a lowered symmetry in the magnetic space group, with the presence of SOC. We define the order parameter, $\boldsymbol{M}=-\sum_{i} \boldsymbol{S}_{i}^{A}+\sum_{i} \boldsymbol{S}_{i}^{B}$, as a linear combination of $\mathrm{Fe}$ spins at $\mathrm{A}$ and $\mathrm{B}$ sites. Using the transformation rules given in Table 【. we analyze, in the thermodynamic free energy, the possible ME coupling terms of the form $\boldsymbol{P} \cdot \boldsymbol{M}^{2}$, which are invariant under symmetry operations.

$$
\begin{aligned}
& F_{\mathrm{ME}}=c_{a a} P_{a} M_{a}^{2}+c_{a b} P_{a} M_{b}^{2}+c_{a c} P_{a} M_{c}^{2}+c_{c a} P_{c} M_{a}^{2} \\
& \quad+c_{c b} P_{c} M_{b}^{2}+c_{c c} P_{c} M_{c}^{2}+c_{a a c} P_{a} M_{a} M_{c} \\
& \quad+c_{c a c} P_{c} M_{a} M_{c}+c_{b a b} P_{b} M_{a} M_{b}+c_{b b c} P_{b} M_{b} M_{c}
\end{aligned}
$$

whereas the dielectric energy is traditionally written as: $F_{\mathrm{DE}}=-\boldsymbol{P}^{2} / 2 \chi$, where the $c_{i j}, c_{i j k}$ coefficients and $\chi$ (set as 1 in what follows) are constants. The minimum of $F=F_{\mathrm{ME}}+F_{\mathrm{DE}}$ occurs when $\partial F / \partial P_{a}=\partial F / \partial P_{b}=$ $\partial F / \partial P_{c}=0$, so that $\boldsymbol{P}$ is obtained. After assuming a simultaneous rotation of Fe spins in the $b c$ plane by an angle $\theta$ with respect to the $c$ axis, setting $\boldsymbol{M}=$ $M(0, \sin \theta, \cos \theta)$, we derive

$$
\begin{aligned}
& P_{a}(\theta)=\frac{M^{2}}{2}\left(-c_{a b}+c_{a c}\right) \cos 2 \theta+\frac{M^{2}}{2}\left(c_{a b}+c_{a c}\right), \\
& P_{b}(\theta)=\frac{M^{2}}{2} c_{b b c} \sin 2 \theta, \\
& P_{c}(\theta)=\frac{M^{2}}{2}\left(-c_{c b}+c_{c c}\right) \cos 2 \theta+\frac{M^{2}}{2}\left(c_{c b}+c_{c c}\right) .
\end{aligned}
$$

Remarkably, the $\theta$-dependence perfectly agrees with the experimentally observed property, $P_{a} \propto \sin ^{2} \theta$. [9] Incidentally, we note that other ME systems often need two or more antiferromagnetic order parameters to cause ME effects [21], whereas here only one magnetic order parameter $\boldsymbol{M}$ is relevant.

Now, selecting the $\theta$-dependent terms from Eq.(1), we obtain;

$$
F_{\mathrm{ME}}(\theta)=\frac{M^{4}}{2}\left(a_{\mathbf{P}} \cos 2 \theta+b_{\mathbf{P}} \sin 2 \theta\right),
$$

where $a_{\mathbf{P}}=\left(-c_{a b}+c_{a c}\right) P_{a}+\left(-c_{c b}+c_{c c}\right) P_{c}$ and $b_{\mathbf{P}}=$ $c_{b b c} P_{b}$. Here, the $\theta$-dependence of $F_{\mathrm{ME}}$ is nothing but the MAE, i.e., we obtained an expression for the MAE as a function of $\boldsymbol{P}$. This is consistent with what already proposed by Rado et. al., where they assumed the electric field $\boldsymbol{E}$-dependence of the MAE coefficient $K_{b}^{\prime}$. [9] Our additional advantage here is that we obtain the $\theta$ dependence of $\boldsymbol{P}$ starting from the polar $C c$ symmetry, which was unknown at that time. Also note that none of the experimentally proposed centrosymmetric structures (e.g., $P 2 / c$ or Pnma) allows for $\boldsymbol{M}$-induced $\boldsymbol{P}$, because the inversion symmetry is not broken by the ferrimagnetic order (all Fe-B spins are parallel). Our findings related to $\mathrm{ME}$ effects are therefore an indirect confirmation that, among those that were experimentally put forward, the $C c$ symmetry is the ground state of the CO-phase.

DFT calculations - In order to quantitatively evaluate the $\boldsymbol{P}(\theta)$ behavior and to investigate the microscopic mechanism, DFT calculations were performed using the VASP 22] code. Starting from a previous study by Jeng et al. 16], we used their optimized $C c$ structural parameters and the GGA $+U[23]$ approach, with $U=4.5 \mathrm{eV}$ and $J=0.89 \mathrm{eV}$ for Fe- $d$ state $\left(\mathrm{Fe}-3 d^{6} 4 s^{2}\right.$ electrons are treated as valence). The $2 \times 2 \times 1$ Monkhorst-Pack $k$-point grid was used; other details are the same as in Ref. 5 . A collinear ferrimagnetic configuration was set, with all $\mathrm{Fe}-\mathrm{B}$ (Fe-A) sites as up-spin (down-spin) sites. By introducing SOC self-consistently, we evaluated both $i$ ) the MAE through the total-energy change and ii) the ME 


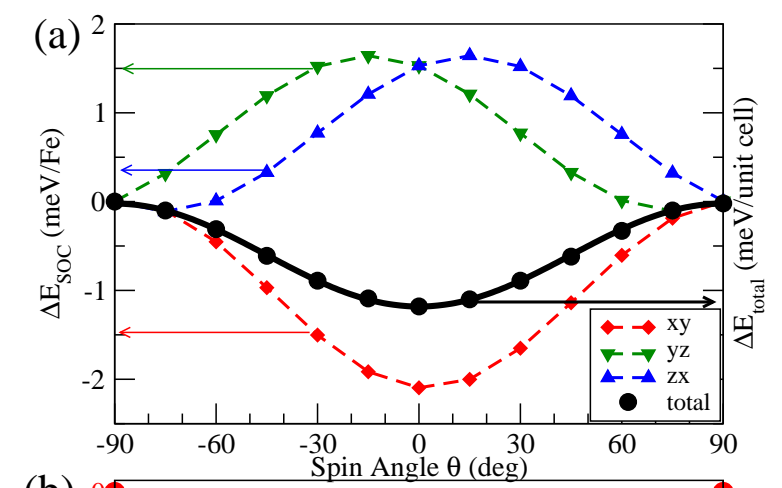

(b)

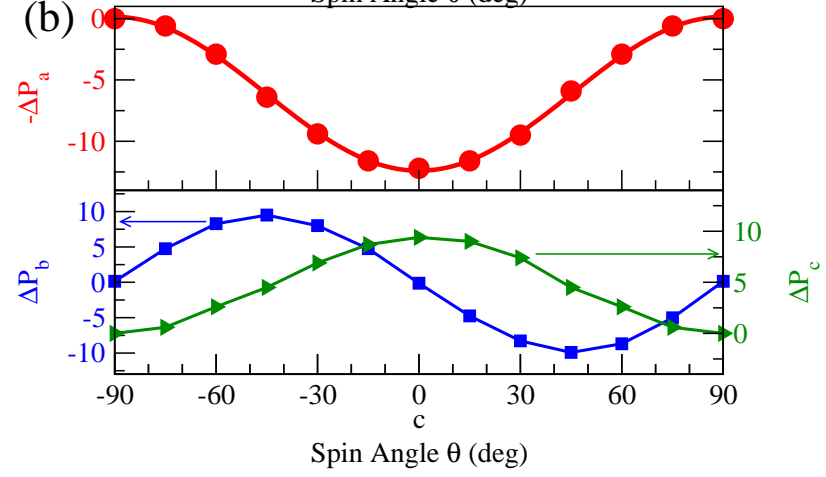

FIG. 2: DFT results: (a) Change in on-site SOC energy $\left(E_{\mathrm{SOC}}\right)$ and total energy $\left(E_{\text {total }}\right)$ versus collinear spin direction $(\theta)$ in the $b c$ plane with respect to the $b$ axis. The label $x y / y z / z x$ denotes the each representative $\mathrm{Fe}^{2+}-\mathrm{B}$ site. (b) Change in $\boldsymbol{P}\left(\mu \mathrm{C} / \mathrm{m}^{-2}\right)$ versus $\theta$ with respect to $P_{a}=-3.86$, $P_{b}=0$ and $P_{c}=4.89 \mu \mathrm{C} / \mathrm{cm}^{-2}$ at $\boldsymbol{M} / / \boldsymbol{b}$. In order to compare with experimental results (Ref. 9), the sign of $\Delta P_{a}$ is reversed and fitted to a function of $a_{1} \sin ^{2} \theta+a_{2} \sin ^{4} \theta$, with $a_{1}=1.24 \times 10^{-3}$ and $a_{2}=1.29 \times 10^{-5}$ (red solid line).

effect through the change of $\boldsymbol{P}$ calculated by the Berry phase method, 24] upon rotation of the $\boldsymbol{M}$ direction with respect to the crystalline axes.

As for the MAE, our results are consistent with experimental observations, i.e. of the magnetically hard $\boldsymbol{a}$, intermediate $\boldsymbol{b}$ and easy $\boldsymbol{c}$ axes [9] (with energy differences, $E_{a}-E_{b}=4.2 \mathrm{meV} /$ cell and $E_{b}-E_{c}=1.2$ $\mathrm{meV} /$ cell). Experimentally, the large $a b$-plane magnetic anisotropy is well known and used for the $H$-field cooling technique, labeled "magnetic annealing", which is generally performed to grow large single-domain crystals below $T_{\mathrm{V}}$.9] Following the procedure of Ref. 11, the "global" MAE can be decomposed into the "local" MAE, evaluated by the on-site SOC energy, $E_{\mathrm{SOC}}$ [25] at each site. Figure 2 shows the "global" MAE and some selected "local" MAEs, upon rotation of Fe spins in the $a b$ plane. Each local MAE shows a $\cos 2 \theta$ curve, which can be explained by SOC perturbation theory. On $\mathrm{Fe}^{3+}\left(d^{5}\right)$ ions, a large MAE is not expected, due to the quenched orbital states; on the other hand, on $\mathrm{Fe}^{2+}\left(d^{6}\right)$ ions, we can apply the same discussion used for the $\mathrm{Co}^{3+}\left(d^{6}\right)$ ion in $\mathrm{BiCoO}_{3}[26$. The perturbation theory predicts that, when the $d_{x y}^{\downarrow}$ orbital is occupied, the single site MAE is given by $E_{\mathrm{MAE}} \simeq \lambda^{2} / 2\left(1 / \Delta_{\mathrm{JT}}-4 / \Delta_{e_{g}}\right) \cos 2 \theta$, where $\lambda$ is the SOC constant, $\Delta_{\mathrm{JT}}$ is the JT splitting energy, $\Delta_{e_{g}}$ is the energy splitting between $(y z, z x)^{\downarrow}$ and $e_{g}^{\uparrow}$ states, and $\theta$ is the spin polar angle, giving energy minimum at $\theta=0$ $(\boldsymbol{S} / / \boldsymbol{z})$. Our results therefore show that the local magnetic easy axis is perpendicular to the occupied orbital plane (the latter being a hard plane); in other words, the local easy axis at $x y / y z / z x$ site is the $z / x / y$ axis, respectively. Indeed, in Fig. 2 the $x y$-labeled MAE comes from the $x y$ site, showing $\cos 2 \theta$ curve with the minimum at $\boldsymbol{M} / / \boldsymbol{z}(\simeq \boldsymbol{c})$, whereas MAE from $y z$ and $z x$ site shows maximum nearby. The "global" MAE is the composition of these local MAEs, based on a delicate balance of (8xy, $4 y z, 4 z x)$ OO set; the subtle deviation of the local easy axis away from lattice vectors, due to the small tilting of the orbitals, also affects the final result.

As for the focus of the paper, i.e. ME effects, we indeed obtained $\boldsymbol{P}^{\mathrm{ME}}(\theta)$ (cfr Fig[2(b)), showing excellent agreement both with what expected from Landau theory in Eq2 and with experimental results. Unfortunately, the experimental size of $P^{\mathrm{ME}}$ has never been reported so far, so that our comparison cannot be quantitative. In our simulations, we got $P^{\mathrm{ME}} \lesssim 20 \mu \mathrm{C} / \mathrm{m}^{-2}$. This is roughly $10^{3}$ times smaller than $P^{\widetilde{\mathrm{CO}}} \simeq 5 \mu \mathrm{C} / \mathrm{cm}^{-2}$ and even one order of magnitude smaller than $P^{\mathrm{ME}}=800 \mu \mathrm{C} / \mathrm{m}^{-2}$ in a representative $\mathrm{ME}$ system, $\mathrm{TbMnO}_{3}$ [12]; however, it should be large enough to be measured, comparable with observed $P^{\mathrm{ME}}=5 \mu \mathrm{C} / \mathrm{m}^{-2}$ in $\mathrm{RbFe}\left(\mathrm{MoO}_{4}\right)_{2}$. 27] Notably, the $P_{b}$ trend put forward an exciting perspective: its sign can be switched by a $90^{\circ}$-rotation of the magnetic field (from $-45^{\circ}$ to $45^{\circ}$ in Fig. 2(b)), pointing to a magnetic control of ferroelectric polarization. 28.

In order to understand the mechanism driving ME effects, let us examine how Fe- $d$ orbital states are affected by the spin rotation in the $b c$ plane. Table $\amalg$ summarizes

TABLE II: $3 d$-orbital coefficients (in percentage, with spin states summed up) of the highest occupied $d$ orbital and Fe- $d$ occupancy $n_{d}$ on $\mathrm{Fe}^{2+}$ B12 site with different SOC enhancement factors $\lambda(0=$ without SOC, $\times 1=$ with standard SOC, and $\times 10=$ with the SOC term 10 times enhanced) for different $\boldsymbol{M}$ directions.

\begin{tabular}{|cc|ccccc|c|}
\hline$\lambda$ & $\boldsymbol{M}$ & $x y$ & $y z$ & $z x$ & $3 z^{2}-r^{2}$ & $x^{2}-y^{2}$ & $n_{\mathrm{d}}$ \\
\hline 0 & - & 98.85 & 0.02 & 0.37 & 0.76 & 0 & 6.097 \\
\hline$\times 1$ & $\boldsymbol{M} / / \boldsymbol{b}$ & 97.97 & 0.4 & 0.45 & 0.78 & 0.41 & 6.083 \\
$\times 1$ & $\boldsymbol{M} / / \boldsymbol{c}$ & 98.25 & 0.4 & 0.42 & 0.82 & 0.1 & 6.083 \\
\hline$\times 10$ & $\boldsymbol{M} / / \boldsymbol{b}$ & $\mathbf{5 1 . 7 4}$ & $\mathbf{1 7 . 2 5}$ & $\mathbf{1 6 . 8 2}$ & 1.94 & 12.24 & $\mathbf{6 . 1 0 8}$ \\
$\times 10$ & $\boldsymbol{M} / / \boldsymbol{c}$ & $\mathbf{7 3 . 8 2}$ & $\mathbf{7 . 9 2}$ & $\mathbf{7 . 7}$ & 0.1 & 10.46 & $\mathbf{6 . 0 9 7}$ \\
\hline
\end{tabular}

the coefficients of a linear combination of $d$-orbital states for the highest occupied $d$ level [29] and the Fe- $d$ occupancy at Fe-B12(xy) site when $\boldsymbol{M} / / \boldsymbol{b}$ and $\boldsymbol{c}$. To highlight the effects of SOC, three calculations were done (keeping the ions fixed): (i) without SOC term (i.e. where the spin direction doesn't affect the orbitals), (ii) with standard SOC term, and (iii) with SOC artificially enhanced by a factor of 10 . At first glance, $d_{x y}$ shows the largest component in all cases. In the extreme case with 10-times-enhanced SOC, $d_{y z}$ and $d_{z x}$ orbitals have relatively large components, increasing to about $17 \%$ when $\boldsymbol{M} / / \boldsymbol{b}$, compared to only $\sim 8 \%$ when $\boldsymbol{M} / / \boldsymbol{c}$. This result reflects the "inverse effect" of the perturbation theory 
discussed above, i.e. the orbital plane of the partly filled minority-spin state tends to be perpendicular to the imposed spin direction, despite the fact that the structural JT distortion would favor a $d_{x y}$ state. The SOC also changes the occupancy of $d$ electrons according to the spin direction. Although the change is negligibly small in standard SOC calculation, it is probably responsible for the slight change in $\boldsymbol{P}^{\mathrm{ME}}(\theta)$. Considering the above evidences, we propose the following scenario for magnetoelectricity: i) SOC changes the shape of $t_{2 g}^{1 \downarrow}$ orbital when $M$ is rotated (as shown in Fig प(b)). ii) this in turn results in the anisotropic hybridization of the Fe- $d$ state with surrounding $\mathrm{O}-p$ states, so that the gravity center of the $d$-electron (i.e. the Wannier center) is slightly shifted in one direction. iii) On the B12 site, the effect doesn't cancel out due to the lack of inversion-related ion in the unit cell, so that a purely electronic contribution appears in the net $\boldsymbol{P}^{\text {orb }}$. In other words, the fact that the underlying $C c$ symmetry lacks inversion is a necessary ingredient for ME effects to emerge.

Conclusion. - We have shown that the origin of the ME effect in magnetite is "orbital-related" and $\boldsymbol{P}^{\text {orb }}$ is induced by the on-site SOC term. This is clearly different from common conventional mechanisms (reviewed in Ref.[3] ), which invoke inter-site spin interaction, such as magneto-striction and DM interaction, in turn often connected to non-collinear spin-configurations. Rather, magnetoelectricity in magnetite emerge in a fully-collinear spin state with large magnetization, pointing to an easier control of ferroelectric properties via magnetic fields. We also note that the on-site $\boldsymbol{P}^{\text {orb }}$ is not cou- pled with "structural" effects, such as piezo-electric or piezo-magnetic. Rather, the main ingredient which allows magnetite to be ME-active is basically the polar OO pattern of unfilled $t_{2 g}$ states. Indeed, no ionic displacements are involved in the rise of $\mathrm{H}$-induced polarization and, in this sense, we can label this novel ME mechanism as purely driven by electronic (charge and orbital) degrees of freedom. Remarkably, our predicted trends show that the long-sought full control of polarization via a magnetic field can be achieved, with $P_{b}$ switching sign upon a $90^{\circ}$ rotation of $\mathrm{H}$-field. We finally recall that the polar $\mathrm{OO}$ is stabilized together with the polar $\mathrm{CO}$ in the $C c$ structure, through the $\mathrm{CDW}$ instability coupled with the Fermi-surface nesting below $T_{\mathrm{V}}$. 30] These peculiar properties make magnetite rather unique; however, we propose at least one candidate where the phenomenology might be similar: $\mathrm{K}_{0.6} \mathrm{FeF}_{3}$, 31] showing a non-centrosymmetric $\mathrm{CO} / \mathrm{OO}$ pattern and where $\mathrm{ME}$ effects should be explored, to confirm the existence of a novel class of OO-induced magnetoelectrics.

\section{Acknowledgments}

KY thanks A. Tanaka, T. Shishidou, T. Oguchi, T. Kimura, C. Ederer and M. Angst for fruitful discussions. The research leading to these results has received funding from the EU Seventh Framework Programme (FP7/2007-2013) under the ERC grant agreement $n$. 203523-BISMUTH. Computational support from Caspur Supercomputing Center (Rome) is also acknowledged.
[1] S. W. Cheong, and M. Mostovoy, Nature Materials 6, 13-20 (2007).

[2] S. Picozzi, and C. Ederer, J. Phys.: Cond. Mat. 21, 303201 (2009).

[3] M. Fiebig, J. Phys. D: Appl. Phys. 38 R123 (2005).

[4] M. Alexe, et al, Advanced Materials 21, 4452 (2009).

[5] K. Yamauchi, T. Fukushima, and S. Picozzi, Phys. Rev. B 79, 212404 (2009).

[6] $\mathrm{LuFe}_{2} \mathrm{O}_{4}$ was originally proposed as a prototype of this class of ferroelectrics [7], but turned out to be antiferroelectric [8] later on.

[7] N. Ikeda, et al. Nature 436, 1136 (2005).

[8] M. Angst, et al. Phys. Rev. Lett. 101, 227601 (2008).

[9] G. T. Rado, and J. M. Ferrari, Phys. Rev. B 125166 (1975).

[10] M. Mostovoy, et al. arXiv: 1004.2070.

[11] K. Yamauchi, and S. Picozzi, Physical Review B 81, 024110 (2010).

[12] T. Kimura, et. al. Nature (London) 426, 55 (2003).

[13] E. J. W. Verwey, Nature 144327 (1939).

[14] J. García, and G. Subías, J.Phys.: Cond. Mat. 16 R145 (2004).

[15] J. M. Zuo, J. C. H. Spence, and W. Petuskey, Phys. Rev. B 428451 (1990).

[16] H.-T. Jeng, G. Y. Guo, and D. J. Huang, Phys. Rev. B
74195115 (2006).

[17] In the lower half of unit cell, another inversion-unpaired $\mathrm{Fe}^{2+}\left(\mathrm{B} 12\right.$ ') $-\mathrm{Fe}^{3+}$ (B13') sites form the electric dipole. [5].

[18] In this paper, the suffixes of $d_{x y}, d_{y z}$ and $d_{z x}$ are always consistent with the $(x, y, z)$ vectors shown in Fig 1 We denote the corresponding $\mathrm{Fe}^{2+}$ site as simply $x y, y z$ and $z x$ site, respectively.

[19] C. Jia, et. al. Phys. Rev. B 76, 144424 (2007).

[20] L. D. Landau, and E. M. Lifshitz, Statistical Physics, Part I (Pergamon Press, Oxford, 1980).

[21] S. Picozzi, et. al. Phys. Rev. Lett. 77, 227201 (2007), and the auxiliary material, E-PRLTAO-99-004748.

[22] G. Kresse, and J. Furthmüller, Phys. Rev. B 54, 11169 (1996).

[23] V. I. Anisimov, F. Aryasetiawan, and A. I. Lichtenstein, J. Phys.: Cond. Mat. 9, 767 (1997).

[24] R. D. King-Smith, and D. Vanderbilt, Phys. Rev. B 47, 1651 (1993);

[25] The SOC energy, $E_{\mathrm{SOC}}=\left\langle\frac{1}{c^{2}} \frac{1}{r} \frac{d V}{d r} l \cdot s\right\rangle$ is integrated in each atomic sphere, where $c$ is the velocity of light, $r$ is the radial distance in each atomic sphere, $V$ is the effective potential as a function of $r$, and $l$ and $s$ are respectively orbital and spin operators. Also see Ref. 11.

[26] Y. Uratani, T. Shishidou, and T. Oguchi, J. Phys. Soc. Jpn. 78, 084709 (2009). 
[27] M. Kenzelmann, et. al. Phys. Rev. Lett. 98, 267205 (2007).

[28] From the symmetry point of view, $P_{b}$ is not allowed, due to the $c$-glide in the $C c$ space group. When considering the magnetic space groups, the $c$-glide is kept as $c(T c$; with time reversal) when $\boldsymbol{M}$ is parallel to $\boldsymbol{b}$ (to $\boldsymbol{c}$ ). However, when $\boldsymbol{M}$ is rotated between $\boldsymbol{b}$ and $\boldsymbol{c}$, no symmetry exists (except for the identity $E$ ), so that any direction of $\boldsymbol{P}$ is allowed, including $P_{b}$ which is purely SOC induced.

[29] The coefficients of $d$-orbitals are obtained by diagonalizing an effective on-site Hamiltonian: $H_{i j}=1 / 2 \delta_{i j}-$ $|i\rangle\langle j| U_{\text {eff }}$, where $|i\rangle\langle j|$ is $10 \times 10$ density matrix (5 orbitals $\times 2$ spins $)$ used in GGA $+U$ procedure and $U_{\text {eff }}$ is an effective on-site Coulomb parameter, described as $U_{\text {eff }}=U-J=3.61 \mathrm{eV}$ by $\mathrm{GGA}+U$ parameters. The occupancy is obtained by the trace of density matrix.

[30] J. P. Wright, J. P. Attfield, and P. G. Radaelli, Phys. Rev. Lett. 87266401 (2001).

[31] K. Yamauchi and S. Picozzi, Phys. Rev. Lett. 105107202 (2010). 